References

Christensen, P. \& Koldbaek, I. (1982) EEG monitored ECT. British Journal of Psychiatry, 141, 19-23.

JONES, E. (1988) Inadequate seizures with electroconvulsive therapy. British Journal of Psychiatry, 153, 264-265.

SIR: Dr Jones has suggested we have misquoted Christensen \& Koldbaek (1982). Not so. Perhaps the insertion of two commas might help her understand the sentence "... found $43 \%$ of fits inadequate, in terms of EEG signs, when judged by clinical observation alone".

It is widely accepted that EEG monitoring provides the most accurate measure of seizure activity. For better or worse, we used it as the standard by which we judged clinical recognition. Thus, to be consistent, no EEG fit/clinical fit is interpreted as clinical misdiagnosis of a seizure.

A tautology is "saying the same thing twice over in different words" (Oxford English Dictionary). We fail to see how changing the definition of an EEG seizure and looking again at clinical seizures is tautological.

Dr Jones has read correctly our paper when she says that in the vast majority of cases EEG and clinical methods do agree. Disagreement in $8 \%$ of unilateral fits suggests to her that EEG monitoring of unilateral ECT is unwarranted. That is debatable. Ten of 17 patients given unilateral ECT had at least one EEG monitored fit of less than 25 seconds. If fit length is therapeutically crucial, we repeat our suggestion that the case for routine EEG monitoring is then greatly strengthened.

Dr Jones has encountered practical difficulties in EEG monitoring. Such difficulties may exist, but can be overcome. ECT is an important treatment, and it behoves all of us to examine closely our clinical practice.

Department of Clinical Research

Robin G. MCCREADIE

Crichton Royal Hospital

Dumfries DG1 4TG

\section{Depression in attempted suicide}

SIR: Ennis et al (Journal, January 1989, 154, 41-47) state that our study of patients who had attempted suicide (Goldney et al, 1981) "reported the highest prevalence of depression". This is demonstrably not so, as two of the five other studies they quote in their Table II found an even higher prevalence of depression.

In considering the different sub-types of depression, there appear to be data missing from their Table I. Dr Ennis et al noted that our figure for the delineation of endogenous depression, $36 \%$, was higher than those for affective disorder or endogenous depression detected by others, which were of the order of $10-17 \%$. Their results are also appreciably greater than those previous results, but they have chosen to report them as indicating that "only" $31 \%$ were diagnosed with a major depressive episode.

They correctly acknowledge the problems of nosology of depression, and suggested that, "since only $8 \%$ of the sample met criteria for melancholia, bipolar illness or major depression with psychotic features, the rate of 'endogenous' depression can be assumed to have been low". This appears to be an unnecessarily restrictive assumption. Indeed, their figure of $31 \%$ is not dissimilar to the $36 \%$ of our subjects who were delineated as having "endogenous" depression, based on responses to the Levine-Pilowsky Depression (LPD) Questionnaire (Pilowsky \& Boulton, 1970). At the very least, it can be asserted that the symptoms which contributed to that allocation of diagnosis by the LPD, and which were described in an earlier Australian study (Goldney \& Pilowsky, 1979), are similar to those required for a DSM-III diagnosis of major depression.

The significance of individual symptoms in depression has long been debated. Pollitt (1971) postulated the concept of a "depressive functional shift" to describe the physiological symptoms which delineate depressive illness, and noted that it was an attempt "to find a nucleus of depressive illness; a timeless clinical index which, while being independent of culture and era, could be confidently assessed and communicated". He added that the value of the concept was that "a functional shift, however small, could be useful in distinguishing depressive illness from natural unhappiness". The symptoms employed by Dr Ennis et al to fulfil DSM-III criteria for a major depressive episode and those employed in our study to delineate endogenous depression are consistent with Pollitt's "depressive functional shift".

It is tempting to draw an analogy between angina and myocardial infarction, with the 'functional shift' of symptoms of depression being analogous to angina. Cardiologists have the benefit of electrocardiogram and enzyme changes to delineate the boundary between angina and infarction; psychiatrists as yet have no such instruments to provide precise delineation between the symptoms of the "functional shift' and a depressive illness.

The above points are somewhat speculative. What is not speculative is the manner in which Dr Ennis et al have reported that "only" $31 \%$ of their subjects were diagnosed as having a major depressive episode, despite their figure being higher than a number of 